\title{
Línea base de I+D+i en la Facultad de Ingeniería y la Escuela de Microbiología en el periodo 2008-2012
}

Óscar Molina ${ }^{1}$

Alberto Urbina ${ }^{2}$

\section{RESUMEN}

Los nuevos cambios que se están produciendo en la economía mundial están llevando a considerar al conocimiento como el elemento básico para generar valor y competitividad. En la nueva dinámica de la sociedad del conocimiento, las instituciones cada vez más se encuentran interesadas en definir, valorar, controlar y gestionar el capital intelectual como elemento fundamental para la competitividad dentro del actual contexto socioeconómico.

Indudablemente, crear conocimiento será la clave para sostener la ventaja competitiva de cualquier institución en el futuro, ya sea de producción de bienes o prestación de servicios. A partir de estas nuevas teorías, fundamentadas en la sociedad del conocimiento, es que en los últimos años se han generado muchos trabajos teóricos sobre la importancia del capital Intelectual.

El modelo del capital intelectual desarrollado a partir de indicadores, es muy útil en las instituciones de educación superior (IES), ya que es una herramienta de apoyo que permite determinar y establecer una mejor valoración de las capacidades que agregan valor a la institución a partir de los activos intangibles que se generan producto de las actividades de I+D+i (investigación científica, desarrollo tecnológico e innovación).

El modelo del capital intelectual para las universidades establece tres factores claves para su comprensión: a) capital humano, b) capital estructural y c) capital relacional. A partir de estos factores, la investigación propone construir una línea base que hace posible mostrar la realidad de la I+D+i en la Facultad de Ingeniería y

\footnotetext{
1 Profesor universitario, Dirección de Investigación Científica y Posgrado, UNAH:investigacionunah.pi.innov.om@gmail.com; oscarmolina.unah@yahoo.com.

2 Jefe del Depto. de Propiedad Intelectual e Innovación, Dirección de Investigación Científica y Posgrado, UNAH: investigacionunah.pi.innov.au@gmail.com; innovagroseire21@yahoo.com.
} 
la Escuela de Microbiología como población meta. La Escuela de Microbiología se incorpora como la población modelo. La línea base tiene corte transversal y abarca el periodo 2008-2012.

A partir de la investigación se demostró que la mayor productividad, en términos de proyectos de investigación científica realizados en el periodo 2008-2012, corresponde a la Escuela de Microbiología con cuarenta y ocho (48) proyectos; con un promedio anual de 9.6 proyectos. Las carreras de la Facultad de Ingeniería, en comparación a la Escuela de Microbiología, tienen una baja producción científica. Ingeniería Industrial con diecinueve (19) proyectos de investigación y la Carrera de Ingeniería Civil con diecisiete (17), son las que más predominaron en la ejecución de proyectos. Ingeniería Química fue la carrera con menor participación, ejecutando un (1) proyecto de investigación.

Palabras claves: investigación científica, desarrollo tecnológico, innovación, capital intelectual, factor humano, factor relacional, factor estructural.

\section{ABSTRACT}

The new changes produced in global economy are considering knowledge as the basic element for generating value and competitiveness. Recently in the society of knowledge, the institutions are interested in defining, assessing, controlling and managing intellectual capital as a key for competitiveness within the socioeconomic context element.

Undoubtedly, the creation of knowledge will be the key for the maintenance of a competitive advantage in any institution, whether it relates to the production of goods or of services. From these new theories based on the knowledge society, $d$ many theoretical scripts have identified the importance of the Intellectual Capital.

The model of intellectual capital has been developed from indicators, which are very useful in higher education institutions (HEls). This model functions as a support tool for determining a better assessment of the capabilities that add value to the institution, primarily those that derive from intangible assets as a product of $I+D+i$ (scientific research, technological development and innovation.)

The model establishes three keys for understanding the factors: a) human capital, b) 
structural capital c) relational capital. From these factors, the research proposes to build a base line that will show the reality of $I+D+i$ in the Faculty of Engineering and the School of Microbiology as the target population. The School of Microbiology will be incorporated as the model population. The base line has a cross section design and it will cover the period for the following years 2008-2012.

From the research an increased productivity was demostrated in terms of scientific research projects conducted from 2008 to 2012. The School of Microbiology showed forty-eight (48) projects; with an average of 9.6 projects per year.

In the Faculty of Engineering the data showed 19 research projects in the area of Industrial Engineering and 17 research projects in Civil Engineering, mostly focusing in project implementation. Chemical Engineering developed just 1 research project.

Keywords: scientific research, technological development, innovation, intellectual capital, human factor, relational factor, structural factor. 


\section{INTRODUCCIÓN}

En los últimos años se ha llevado adelante una serie de trabajos teóricos tendientes a conocer el capital intelectual en las instituciones de educación superior. Algunos especialistas en temas de gestión universitaria, como Susana Darín (2005), destacan la capacidad de contar con un sistema de medición que asegure la mejora continua del proceso de aprendizaje institucional. La particularidad de este enfoque de medición facilita, a través de su interpretación, la identificación y valoración de información sobre los elementos claves de la institución como requerimiento para la toma de decisiones.

En la nueva dinámica de la tercera misión de las instituciones de educación superior se propician herramientas como la del capital intelectual para integrar los conceptos de I+D+i en la gestión institucional y evaluar las capacidades y competencias atinentes a estos ámbitos. Salazar (2005) señala que a través del enfoque del capital intelectual, las entidades de educación superior pueden identificar los puntos fuertes y débiles del proceso, al mismo tiempo la decisión de definir estrategias para llevar a cabo los correctivos y diseñar metodología para una administración eficaz.

El término capital intelectual dentro de las universidades será usado para estudiar todos los activos no tangibles o no físicos de la institución, incluyendo sus procesos, capacidad de innovación, patentes, el conocimiento tácito de sus miembros y su red de colaboradores y contactos. Se puede definir al capital intelectual como el conocimiento que la universidad posee y que crea o puede crear valor en sus procesos de generación y construcción de conocimiento, pero además en la gestión académica; está conformado por todos aquellos actores y elementos constitutivos que generan valor para la institución.

El modelo del capital intelectual para las universidades trata de visualizar las actividades de $1+D+i$ como un proceso y establece tres elementos claves para su valoración: capital humano, capital estructural y capital relacional. En el contexto de las universidades, el capital humano es el conocimiento tácito y explícito de los investigadores y del personal de apoyo no científico. El capital estructural comprende las rutinas y procesos en la universidad, incluyendo la infraestructura. El capital relacional comprende las relaciones y redes de los investigadores, así como de toda la organización.

Un estudio basado en el enfoque del capital intelectual, permite identificar tanto debili- 
dades como fortalezas estructurales o dividuales; a la vez que constituye un estado del arte en cada una de las misiones de las instituciones de educación superior, entendidas como formación, investigación y extensión; por ende, es un mecanismo de control y monitoreo del valor agregado para la gestión de los procesos de l+D+i.

Para propósitos del estudio, trabajar en la arquitectura de una línea base desde el enfoque del capital intelectual, exigió disponer de información adecuada en un periodo de referencia. Para ello, se seleccionó el periodo 2008-2012 y se delimitó el campo de estudio hacia la Facultad de Ingeniería y la Escuela de Microbiología, sin perjuicio de que este pueda ser replanteado hacia otros escenarios de la universidad.

El estudio permitió conocer en detalle la realidad de estas unidades académicas en los ámbitos de la I+D+i, lo cual constituye una primera aproximación para definir estrategias y procedimientos estandarizados que garanticen la obtención de la información requerida para la toma de decisiones.

\section{METODOLOGÍA}

La línea base es un instrumento descriptivo a través del cual, en forma rápida y operativa, es posible aproximarse a la identificación de la realidad estudiada. El análisis es de corte transversal para el periodo de 2008-2012. Los indicadores diseñados surgen de los factores determinados y permiten fehacientemente capturar información relevante, pertinente y útil para tomar decisiones coherentes con las políticas y estrategias institucionales en materia de $1+D+i$.

En la definición de las variables de estudio, la línea base se apoyó en el modelo del capital intelectual para actividades de investigación y desarrollo de las instituciones de educación superior (IES), propuesto por FH JOANNEUM. ${ }^{3}$ Las variables de estudios se establecieron en función del modelo analizado, el cual establece tres factores fundamentales: humano, estructural y relacional.

1. Factor humano. Para fines de la línea base, este indicador se entiende como las personas medidas a través de su nivel académico, experticia y actividades desarrolladas en $\mathrm{I}+\mathrm{D}+\mathrm{i}$.

3 La University of Applied Sciences, estableció el método FH JOANNEUM con el objetivo de ilustrar la manera en que se pueden desarrollar los indicadores para la medición del capital intelectual en las IES en las áreas de investigación y desarrollo, como herramienta clave para la gestión de los activos intangibles en las IES (Kok, 2007). 
2. Factor estructural. Es entendido como todo medio científico tecnológico que contribuye al logro de los objetivos cuantitativos y cualitativos de los planes estratégicos de la institución y por principio son propiedad de esta. Son determinantes en la calidad de los productos, procesos y servicios, sin embargo, por sí solos son irrelevantes, ya que necesitan de la participación de los actores de ciencia y tecnología para su actualización y alto rendimiento.

3. Factor relacional. Son las interacciones de los actores de $++D+i$ de la universidad focalizadas para fines del estudio, como los convenios internacionales y nacionales, congresos organizados, ponencias presentadas, estancias de investigación, etc.

\section{POBLACIÓN Y MUESTRA}

El método de muestreo utilizado fue el aleatorio simple. El procedimiento fue el siguiente:

1. Identificación de las carreras.

2. Elaboración de listados: decanos, jefes de departamentos, coordinadores de carrera y jefes de unidades de investigación.

3. Seleccionar la muestra de los sujetos según carrera.

4. Se estableció como criterio, que en el caso que los sujetos de investigación por cada carrera fuesen 20 , se haría un censo y se investigaría el universo poblacional.

\section{RECOLECCIÓN DE LA INFORMACIÓN}

Se utilizó el instrumento de entrevista semiestructurada para recoger datos cualitativos que ayudaron a valorar la percepción, conciencia, conocimiento, actitudes y prácticas relacionadas a I+D+i y propiedad intelectual. Además, se diseñó una encuesta estructurada y se aplicó a los sujetos bajo el método aleatorio simple, para recopilar información específica en función de las variables de estudio. La encuesta 
(cuestionario) se aplicó de forma impresa y virtual según el grado de dificultad para localizar físicamente a los sujetos de estudio

Gráfico 1. Cantidad de profesores en planilla, según carrera, 2012

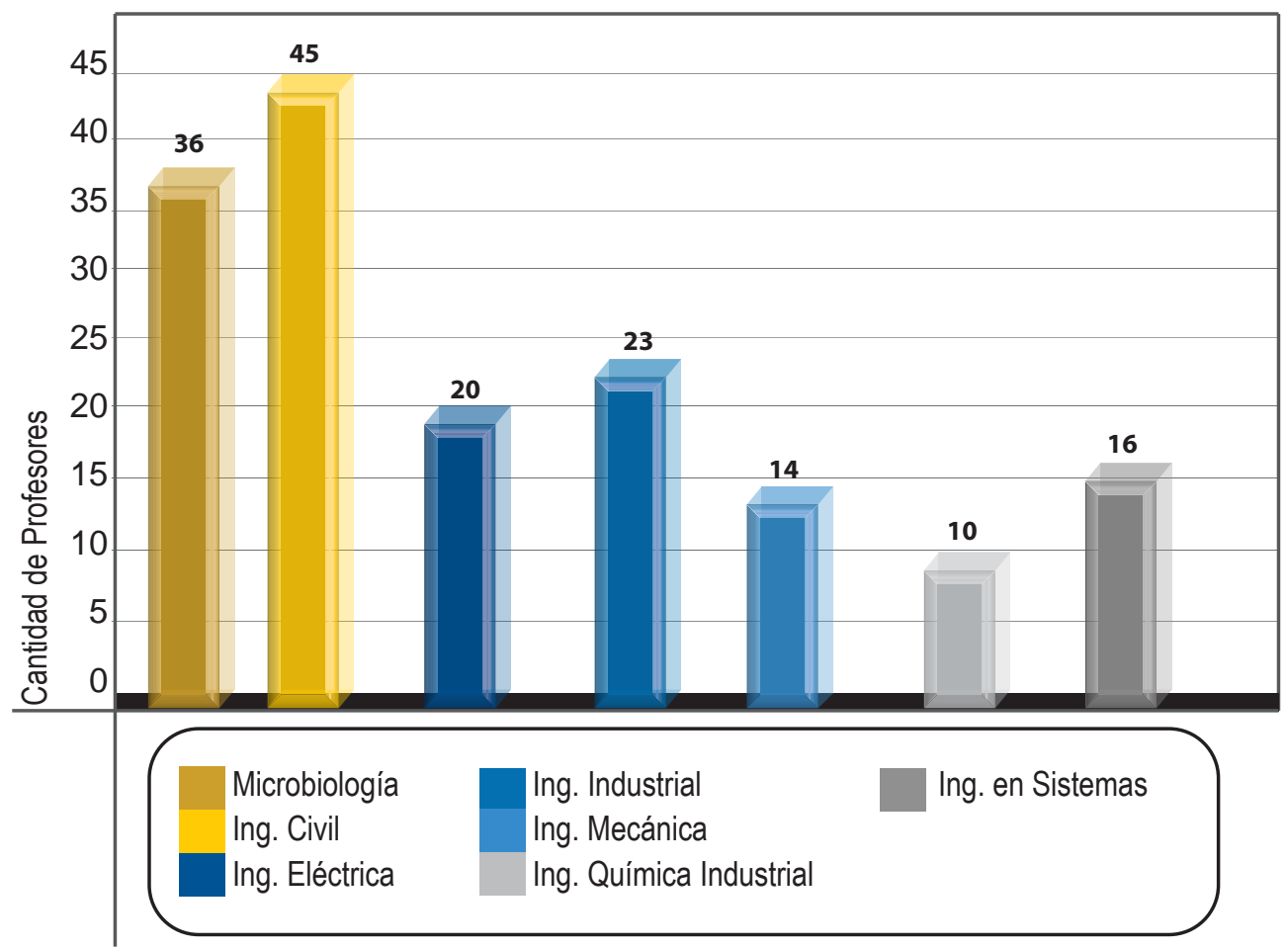

De acuerdo a los datos del gráfico 1, el recurso humano docente de las carreras de la Facultad de Ingeniería y la Escuela de Microbiología se distribuye de la forma siguiente: Ingeniería Civil, cuarenta y cinco (45) profesores; Ingeniería Industrial, veintitrés (23); Ingeniería Eléctrica, veinte (20); Ingeniería en Sistemas, dieciséis (16); Ingeniería Mecánica, catorce (14); Ingeniería Química Industrial, diez (10) y la Escuela de Microbiología, treinta y seis (36).

La planilla más amplia corresponde a la Carrera de Ingeniería Civil con cuarenta y cinco (45) profesores y la más reducida se concentra en la Carrera de Ingeniería Química Industrial con diez (10).

Las cifras anteriores revelan las potencialidades humanas de la Facultad de Ingeniería para ser auténticos centros de generación de conocimientos científicos y tecnológicos, además de cumplir con su función tradicional de la docencia. 
Gráfico 2. Cantidad de profesores que corresponden a la jornada laboral de tiempo completo, 2012.

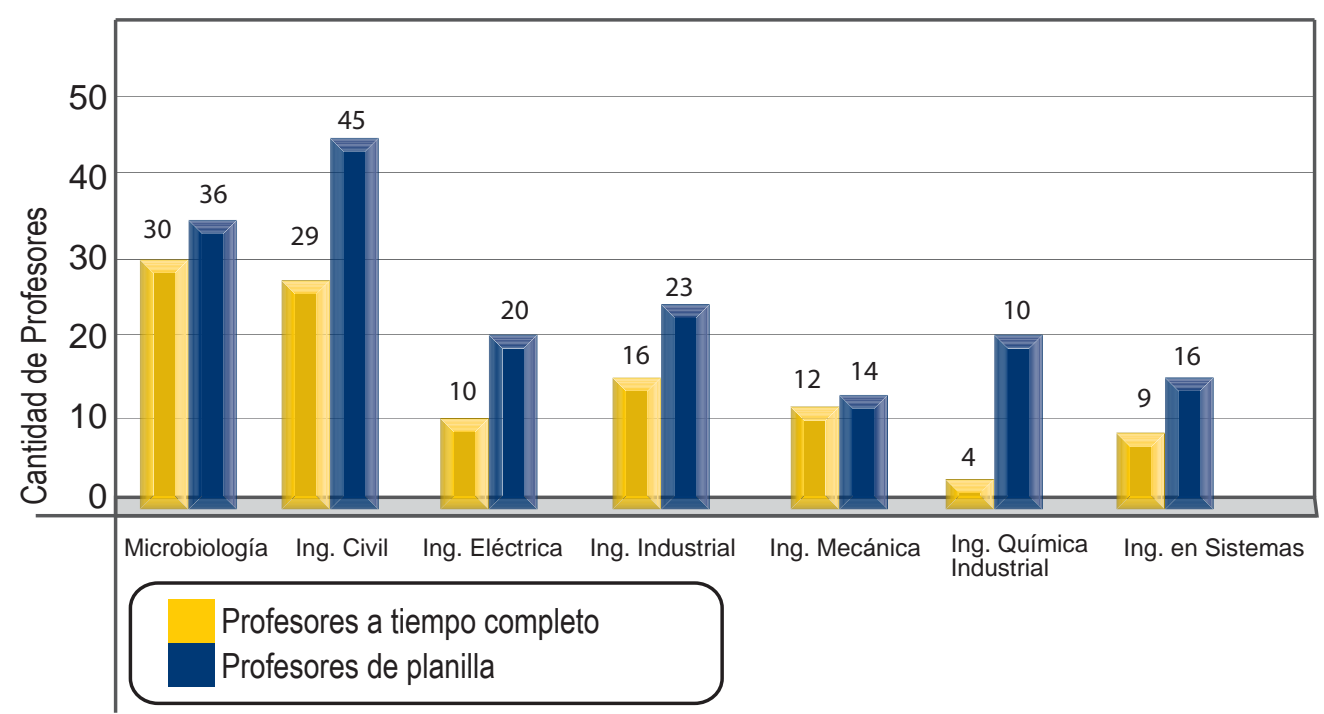

Se puede observar en el gráfico 2 que las carreras que más concentran profesores en la jornada laboral de tiempo completo, son la Escuela de Microbiología con treinta (30) profesores e Ingeniería Civil con (29); siendo esta última la carrera que más profesores a tiempo completo agrupa en la Facultad de Ingeniería. En menor cantidad, se encuentra Ingeniería Industrial con dieciséis (16) profesores e Ingeniería Mecánica con doce (12).

En la carrera de Ingeniería Química Industrial, la mayoría de los profesores no están desempeñando sus actividades o competencias (docencia, gestión, investigación) en la jornada de tiempo completo, dado que solo cuatro (4) de los diez (10) profesores corresponden a esta jornada.

Se encontró en la investigación que Ingeniería Eléctrica e Ingeniería Química Industrial son las que menos disponibilidad de profesores a tiempo completo reportan (menos del $50 \%$ de la planilla). 
Gráfico 3. Cantidad de profesores con doctorado según carrera, 2012

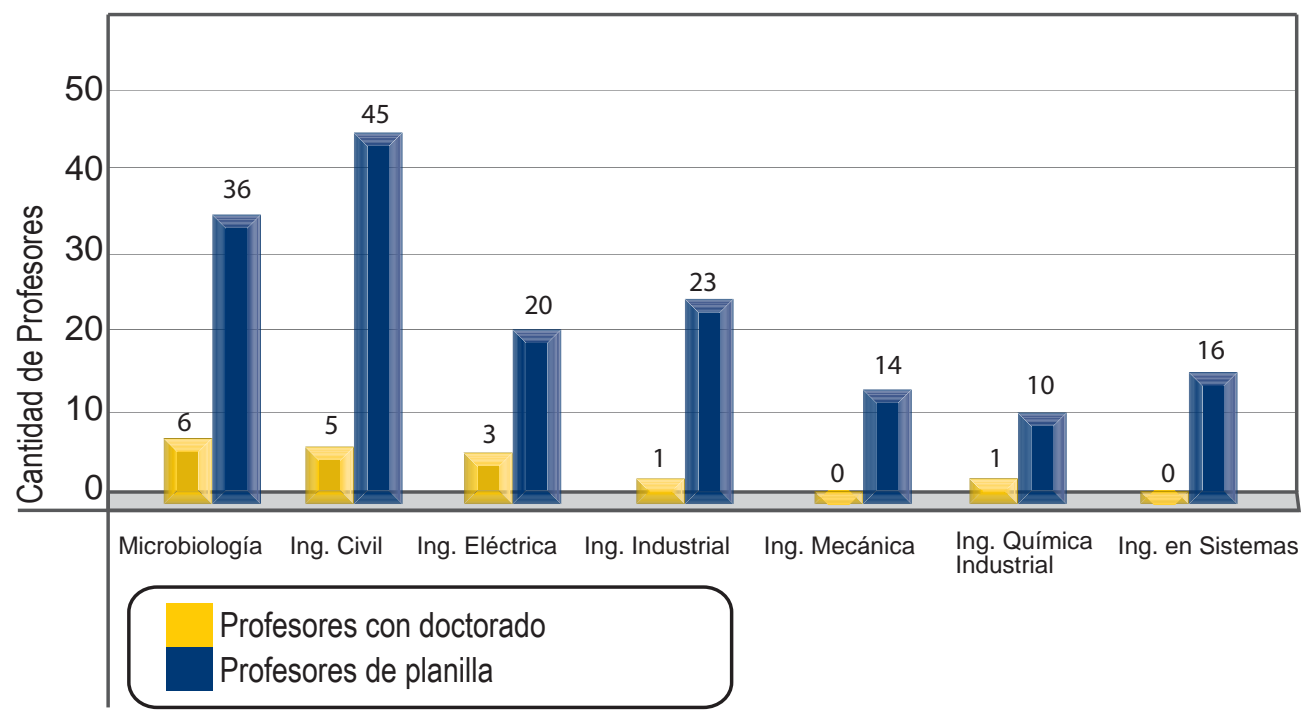

En el gráfico 3 se observa que la Escuela de Microbiología e Ingeniería Civil son las que mayor disponibilidad de profesores con doctorado tienen, seis (6) y cinco (5) profesores, respectivamente.

Ingeniería Mecánica e Ingeniería en Sistemas son las únicas que no tienen profesores con grado académico de doctorado.

Gráfico 4. Cantidad de profesores con maestría según carrera, 2012

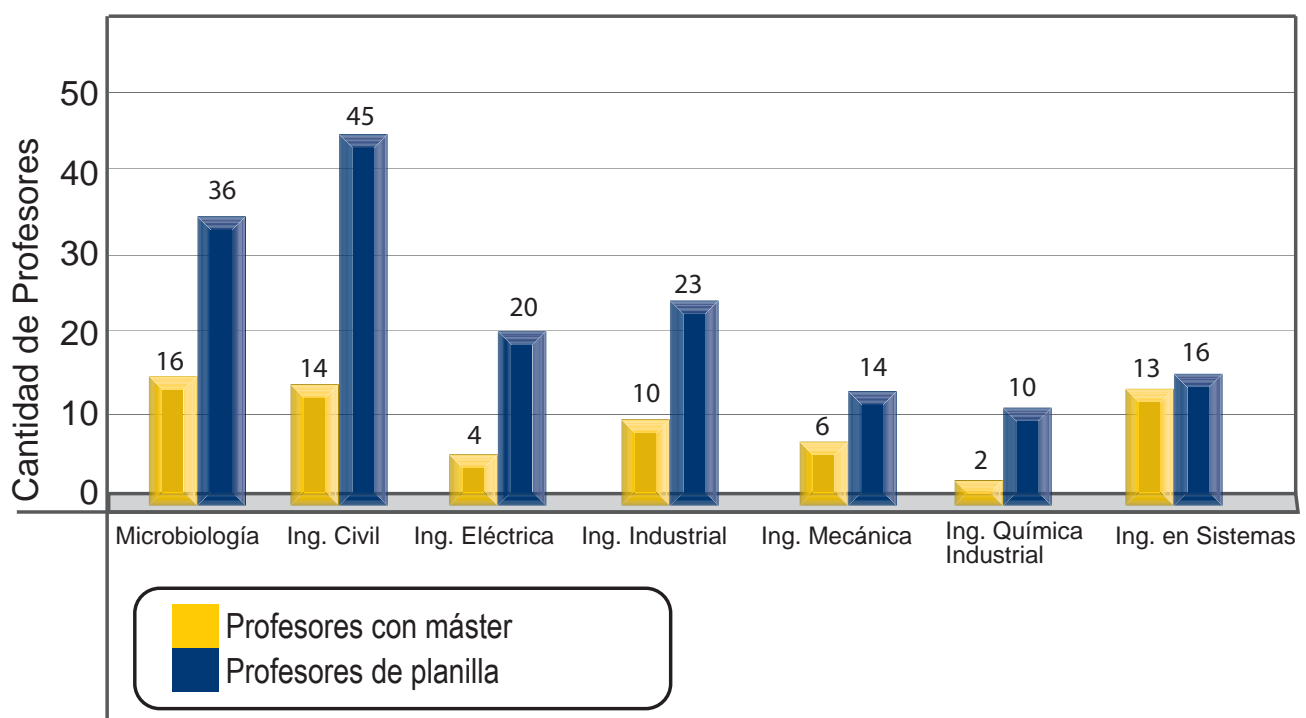


Según el gráfico 4, las carreras que cuentan con más profesores con el grado académico de maestría son: Microbiología con (16) profesores, Ingeniería Civil con (14) e Ingeniería en Sistemas con (13).

Ingeniería Eléctrica tiene cuatro (4) profesores e Ingeniería Química Industrial dos (2), siendo estas las carreras que tienen menos profesores con maestría en sus planillas.

Gráfico 5. Cantidad de investigadores por carrera, 2008-2012

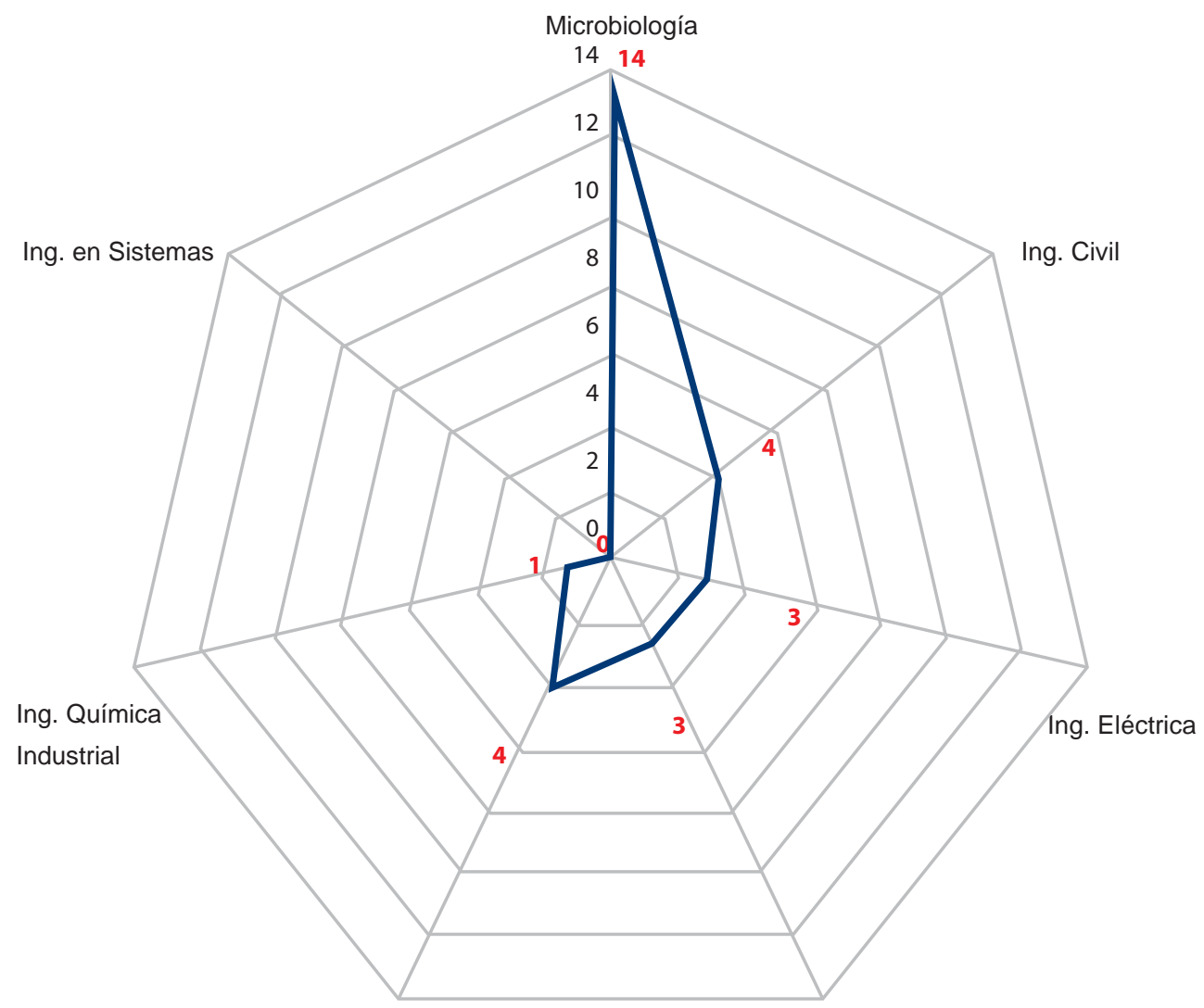

Ing. Mecánica

Ing. Industrial

Profesores que hacen investigación

Como se observa en el gráfico 5, en la Escuela de Microbiología, catorce (14) de los treinta y seis (36) profesores realizaron investigación durante el periodo 2008-2012. En las carreras de la Facultad de Ingeniería se observa una limitada participación de profesores en la investigación, quienes en promedio concentraron entre tres (3) y cuatro (4) profesores por carrera en actividades de investigación. 
A excepción de la carrera de Ingeniería en Sistemas que no registra profesores con proyectos de investigación, todas las demás carreras durante el periodo 2008-2012 realizaron proyectos de investigación científica. Los datos reflejan que la Escuela de Microbiología, en comparación a las carreras de la Facultad de Ingeniera, es en donde más se incorpora la investigación como parte de la carga académica.

Gráfico 6. Productividad científica de las carreras, 2008-2012

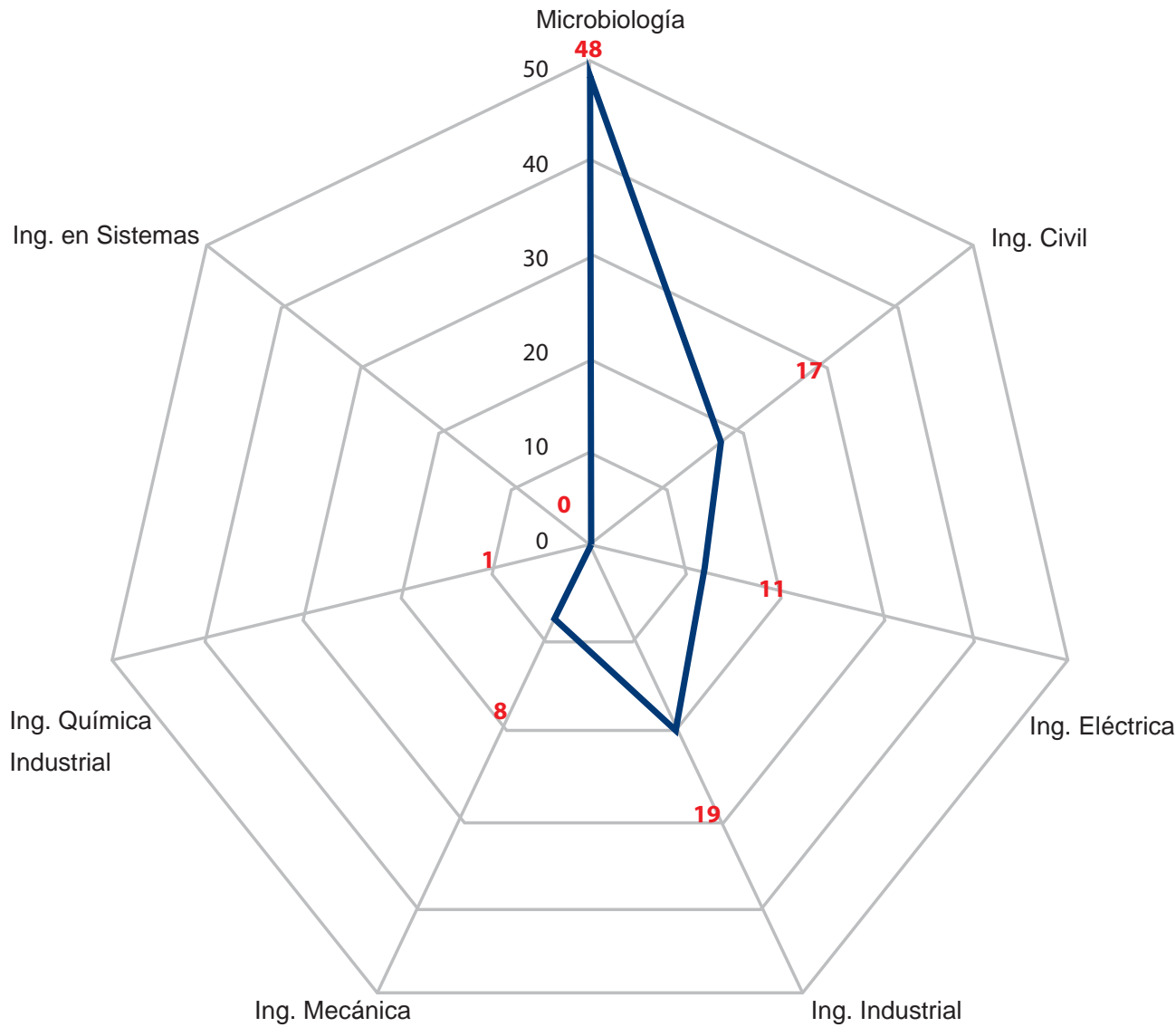

Proyectos de investigación

Como lo muestra el gráfico 6, la mayor productividad en términos de proyectos de investigación científica durante el periodo 2008-2012, corresponde a la Escuela de Microbiología con cuarenta y ocho (48) proyectos ejecutados, con un promedio anual de 9.6 proyectos. 
Las carreras de la Facultad de Ingeniería, en comparación a la Escuela de Microbiología, tuvieron una baja producción científica, encontrándose que las más destacadas son la Carreras de Ingeniería Industrial con diecinueve (19) proyectos de investigación y la Carrera de Ingeniería Civil con diecisiete (17). Las carreras que menor productividad mostraron son Ingeniería Química con un (1) proyecto y la Carrera de Ingeniería en Sistemas que no desarrolló proyectos de investigación.

Gráfico 7. Publicaciones en revistas científicas (indexadas), 2008-2012

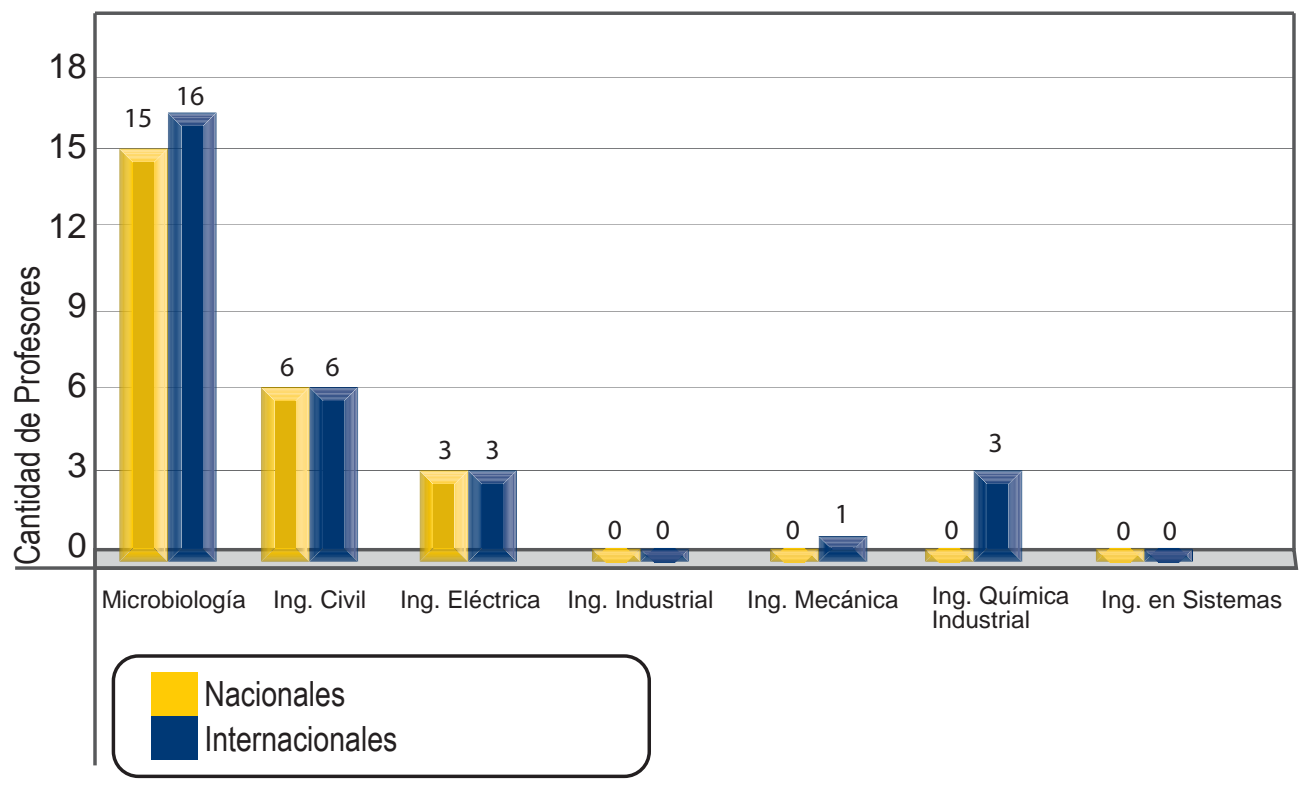

En el grafico 7 se puede apreciar que los profesores de la Escuela de Microbiología presentan la mayor producción de artículos cientíicos, registrando un total de treintaiuna (31) publicaciones: quince (15) en revistas nacionales y dieciséis (16) en revistas internacionales.

En la Facultad de Ingeniería los profesores, en su mayoría, no publicaron artículos científicos en revistas indexadas. La carrera que más publicaciones presentó corresponde a la de ingeniería Civil con doce (12) publicaciones, seis (6) en revistas nacionales y seis (6) en revistas internacionales. Las carreras de Ingeniería Industrial e Ingeniería en Sistemas son las únicas en donde sus profesores no publicaron artículos científicos. 


\section{CONCLUSIONES}

Estos resultados obedecen al análisis global de los factores analizados en la investigación (humano, estructural y relacional):

1. En las Carreras de Ingeniería y en la Escuela de Microbiología, la mayoría de los profesores están adscritos a la jornada de tiempo completo.

2. En la Escuela de Microbiología la investigación científica forma parte importante de la carga académica, lo que ha permitido apalancar y desarrollar una cultura de investigación. En el caso de la Facultad de Ingeniería, el componente de investigación tiene bajo rendimiento, dado que la participación de profesores que hacen investigación por planilla es limitada.

3. En la Escuela de Microbiología la mayoría de los profesores tienen estudios de posgrados, especialmente a nivel de maestría (61.11\%) y el $17 \%$ de los profesores ostentan el grado académico de doctorado.

4. En la Facultad de Ingeniería es notorio que la Carrera de Ingeniería en Sistemas concentra el mayor porcentaje de profesores con formación académica a nivel de maestría (81.25\%). En menor grado, lo tienen la Carrera de Ingeniería Química Industrial e Ingeniería Eléctrica con $30 \%$ y $31 \%$, respectivamente.

5. En la Carrera de Ingeniería Civil el $11 \%$ de los profesores tiene grado académico de doctorado y el $14 \%$ posee grado de maestría.

6. En la Carrera de Ingeniería Eléctrica el $3 \%$ de los profesores tiene doctorado y el $4 \%$ maestría.

7. En la Carrera de Ingeniería Industrial, el $4 \%$ de los profesores tienen grado académico de doctorado y el $43 \%$ el de maestría.

8. En la Carrera de Química Industrial, el $10 \%$ de los profesores tiene doctorado y el $20 \%$ maestría.

9. En la Carrera de Ingeniería Mecánica no tienen doctores, no obstante, el $6 \%$ de los profesores tiene maestría. 
10. En general, en la Facultad de Ingeniería, el $49.4 \%$ de los profesores tiene estudios de posgrado, de los cuales el $9.8 \%$ tiene grado académico de doctor y el $39.6 \%$ de maestría.

11. La mayor productividad en términos de proyectos de investigación científica durante el periodo 2008-2012, corresponde a la Escuela de Microbiología con cuarenta y ocho (48) proyectos ejecutados, con un promedio anual de 9.6 proyectos.

12. En la Facultad de Ingeniería, las carreras de mayor productividad en investigación son: Ingeniería Industrial (19 proyectos) e Ingeniería Civil (17 proyectos) y la de menor productividad es Ingeniería Química Industrial (1 proyecto), ya que Ingeniería en Sistemas no tiene investigaciones.

13. En la ejecución de proyectos interinstitucionales se observa que la Escuela de Microbiología es la que mayor socialización realizó, ya que durante el periodo 2008-2012 participó en veinticinco (25) proyectos asociados con otras organizaciones u universidades.

14. En la Facultad de Ingeniería, las carreras que han tenido actividades de socialización en la realización de proyectos de investigación científica con otras instituciones son: Ingeniería Civil e Ingeniería Química Industrial (2 proyectos cada uno); Ingeniería Eléctrica e Ingeniería Industrial un (1) proyecto cada uno.

15. Durante este periodo, las carreras de la Facultad de Ingeniería no han realizado ninguna organización o ejecución de eventos científicos (congresos).

16. La Escuela de Microbiología, en el periodo 2008-2012, ejecutó dentro de sus actividades académicas dos (2) congresos de investigación científica. No obstante, las carreras de la Facultad de Ingeniería, en este mismo periodo, no llevaron a cabo congresos de investigación científica y tecnológica.

17. En el periodo 2008-2012, la Escuela de Microbiología ha participado en cinco (5) estancias de investigación.

18. Las carreras de la Facultad de Ingeniería no han promovido programas de pasantías. 


\section{RECOMEDACIONES}

1. Elaborar y desarrollar la estrategia de I+D+i en la Facultad de Ingeniería y aprovechar las ventajas en materia de gestión y ejecución de proyectos de investigación de la Escuela de Microbiología.

2. Fortalecer las unidades de investigación existentes y formarlas donde sean necesarias para el desarrollo de I+D+i.

3. Crear y estimular los círculos de innovación tecnológica en las carreras de Ingeniería y la Escuela de Microbiología.

4. Sensibilizar a las autoridades de la Facultad de Ingeniería para que de oficio y como parte de la política académica, se incorpore la investigación científica y tecnológica, la innovación y la transferencia, en su estrategia de desarrollo científico tecnológico.

\section{BIBLIOGRAFÍA}

Acuña, P. (1993). Vinculación universidad - sector productivo. Revista de Educación Superior, 87, 125-150. México.

Clark, B. (1996). Creando universidades emprendedoras en Europa. Revista Valenciana, 21, 373-392.

Contreras, C. (1979). Una ciencia y tecnología para el desarrollo económico y social. Nueva Sociedad, 21, 5-14.

Darín, S. (2005). Gestión del conocimiento: un nuevo enfoque aplicable a las organizaciones y a la universidad. Buenos Aires: Editorial Norma.

Gould, G. (2002). La vinculación como estrategia de desarrollo en las universidades públicas. Baja California: Universidad Autónoma de Baja California.

Kok, A. (2007). Intellectual Capital Management as Part of Knowledge Management Initiatives at Institutions of Higher Learning. The Electronic Journal of Knowledge Management, 5(2), 181-192. Recuperado de: http://www.ejkm.com/volume5/ issue2/p181

Marchetto, M. (2006). La investigación científica y tecnológica en el ámbito de los institutos tecnológicos. Universidad Centroccidental Lisandro Alvarado. Compendium, 16, 57-66. 
Rojas, L. (1991). A propósito de la relación universidad-empresa: aspectos conceptuales y experiencias en la transferencia de la tecnología. Ponencias del IV Seminario Latinoamericano de Gestión Tecnológica. Caracas: ALTEC.

Vargas, J. H. (2004). Generación y aplicación del conocimiento en la formación de los actores. Cuarto Congreso Nacional y Tercero Internacional: "Retos y Expectativas de la Universidad". Guadalajara: Universidad de Guadalajara. 